\title{
Naturaleza y características de los servicios y los contenidos digitales abiertos
}

\author{
Francisco García y Manuel GerTRUdiX*
}

\author{
Propuesto: 21 de febrero de 2011 \\ Evaluado: 20 de marzo de 2011 \\ Aceptado: 25 de marzo de 2011 \\ (Abstracts y palabras clave al final del texto)
}

\section{INTRODUCCIÓN}

Los servicios y contenidos digitales abiertos han configurado un modelo revolucionario de intercambio y producción de información en la Red. La profusión de herramientas y la capacidad de producción, el incremento de acceso y tratamiento de la información, y la multiplicación de posibilidades de participación y creación a través de servicios y sistemas atractivos y sencillos que los caracterizan (Benkler 2006, Carr 2008) ha dinamitado las lógicas de creación, de producción, de distribución, o de consumo de contenidos. De este modo, la emergencia de los servicios digitales abiertos ha propiciado un escenario nuevo en que aparecen fórmulas de participación social, dinámicas y estrategias de intercambio, de apropiación, de reformulación que desconocíamos, al menos con los rasgos y la profundidad que observamos actualmente.

El artículo realiza una aproximación documental cuya finalidad es reflejar cuál es el estado de la cuestión, considerando las principales aproximaciones teóricas realizadas, y cuáles son las categorías claves que permiten dibujar un escenario fiable del mismo. El recorrido, que se circunscribe al estudio de los servicios y contenidos digitales abiertos en el ámbito de la denominada Web 2.0 o Web Social, comienza en la evaluación de aquellas fuentes que analizan el contexto general en el que se desarrollan los servicios y contenidos digitales abiertos, y que denominamos infoentorno, ya que se parte de la premisa de que, por coherencia sistémica y global, los

\footnotetext{
* Francisco García García es Catedrático de Comunicación Audiovisual y Publicidad de la Universidad Complutense de Madrid. (fghenche@gmail.com). Manuel Gertrudis Barrio es Profesor Titular de Comunicación Audiovisual y Publicidad de la Universidad Rey Juan Carlos de Madrid. (manuel.gertrudix@sonidosimaginarios.es). Este artículo es parte de una investigación I+D titulada La construcción de la realidad social en los jóvenes a través de los servicios y contenidos digitales abiertos (Número: CSO20081496/SOCI. Entidad financiadora: Subdirección General de Proyectos de Investigación. Ministerio de Ciencia e Innovación).
} 
rasgos esenciales de estos han de ser congruentes y consistentes con el espacio en el que se dan. Seguidamente, se aborda el estudio de aquellas referencias que atienden al sistema de construcción social de información en la Red, analizando de este, específicamente, cuáles son las dimensiones de la Red que favorecen la producción compartida de información y conocimiento. Por último, nos adentramos en el análisis de las referencias que definen conceptualmente los servicios y contenidos digitales abiertos y se establece una propuesta inicial tipológica (AAVV, Creative Commons España, 2010) de los mismos.

\section{EL NUEVO ESCENARIO DE SERVICIOS Y CONTENIDOS DIGITALES ABIERTOS EN LA RED}

El sistema de construcción social de información en la Red establece un modelo cultural de creación, procesamiento, compartición, intercambio y remixación de información en la web a través de procesos colectivos basados en una especie de "acervo memético en Internet" que procura formas socializadas, cooperativas y/o colaborativas, de organizar, crear y compartir el conocimiento. Para nuestro análisis, esas formas se concretan en dos aspectos esenciales de este escenario: los contenidos y servicios digitales abiertos.

Los contenidos digitales abiertos (CDA) conforman conjuntos de información multimodal, poseen organización y sentido propio, están publicados en la web mediante una aplicación social que permite a los usuarios, su modificación, reutilización, combinación, comentario, recomendación, selección, registro, y cualquier otra operación de re-construcción o remixación por adicción, supresión, yuxtaposición, combinación. Estos, se distribuyen a través de sistemas de licenciamiento de derechos de propiedad intelectual abiertos que permiten, facilitan, y promueven este tipo de uso, como por ejemplo Creative Commons (AAVV, Creative Commons España, 2010) o Color Iuris (AAVV, 2010), y, en general, los sistemas derivados del CopyLeft. Su existencia, y proliferación, está estrechamente relacionada con las capacidades sociotécnicas de la Web Social y especialmente por las posibilidades crecientes de la computación distribuida en la nube.

Los servicios digitales abiertos (SDA) son todos aquellos aplicativos en línea que realizan el proceso y tratamiento de datos en remoto ante la solicitud de un cliente. Emergidos especialmente a partir del año 2001, se fundamentan técnicamente en conceptos como el Cloud Computing, la sindicación, la publicación y administración simplificada, el seguimiento de estándares, etc. En la actualidad, existe prácticamente un servicio digital abierto para todas las funciones básicas y avanzadas de tratamiento de información que ofrecen las aplicaciones de escritorio convencionales, y más allá de estas, el fenómeno mashup (Sonvilla-Weiss 2010) ha propiciado el desembarco de una nueva generación de aplicativos que mixturan propiedades de varias herramientas para crear o configurar nuevas soluciones mediante asociaciones flexibles (loosely coupled) que facilitan el desarrollo relacional (Pisani, Piotet, \& Delclos (2009: 86). 


\section{EL INFOENTORNO COMO ECOSISTEMA GLOBAL}

Las tecnologías de la información y la comunicación hacen posible una utilización plena y expansiva del concepto que McLuhan (1969) definió para referirse al valor de los medios como extensiones de las capacidades humanas, y que otros autores, como Echeverría (2000) han denominado en el ámbito de la red universal digital como "protesicidad". Este carácter extensivo, incremental, permite a los sujetos "ampliar sus sentidos, sus capacidades de cálculo, su memoria, sus capacidades de comunicación y unida a las propiedades de potencialidad, intangibilidad y ubicuidad de la Red" abren el camino hacia potentes prótesis integradas en los cuerpos y en su envoltura artificial.

Desde las bases de la cibernética (Wiener 1985) y a partir del concepto pergeñado por Gibson en un marco de ficción (2004) la evolución de la red Internet y los modelos de intercambio y vivencia que esta ha facilitado, ha ido tejiendo una construcción y un imaginario sobre ese concepto que supone el ciberespacio. Así, mientras para Kevin Hughes se trata del "ambiente de redes globales sostenido por computadoras y por un espacio interactivo en tiempo real en donde los usuarios pueden colaborar, ver y manipular información" (1995: 35) para M. Benedikt representa "un universo paralelo, creado y sustentado por el mundo de las computadoras y las líneas de comunicaciones. Un mundo en donde el tráfico global de conocimientos, secretos, medidas, indicadores y entretenimientos toman la forma de visiones, sonidos, presencias nunca vistas en la superficie de la tierra. El ciberespacio es cuando una página se convierte en una pantalla y ésta en un mundo, en un mundo virtual donde nada se olvida y todo cambia" (Bell 2007) Por su parte, Javier Echevarría, en su obra de análisis de la cibercultura (2000) contempla la Red a través de una metáfora para describirla como una especie de ciudad virtual, una Atenas virtual, que requiere reglas, que necesita criterios de actuación política, para facilitar una articulación social, y, por lo tanto, parte de una visión "intervencionista" del poder en la Red. Raúl Trejo (2006) define la identidad de la sociedad de la información, como construcción social de ese ciberespacio, a través de un conjunto de propiedades que, de un modo u otro, definen con acierto los principales topoi que caracterizan el ecosistema actual de la Red: desigualdad, exuberancia e irradiación de datos y contenidos, omnipresencia, ubicuidad, inmaterialidad, intemporalidad, innovación, volatilidad, multilateralidad, libertad, interactividad, heterogenidad, convergencia, multilinealidad.

\section{LAS LÓGICAS CULTURALES: EL VÉRTIGO DE LA CULTURA SNACK}

El contexto global, y su dimensión glocal (Castell 2009, Guengerich 2010) que nos ofrece el Infoentorno ha generado sus propias dinámicas sociales y culturales. Como señalan diferentes autores (Miller 2007, Verdú 2007) ese cierto vértigo que fomentan los sistemas tecnológicos actuales ha incentivado un proceso de escapismo continuo del sujeto; de un "estar yendo" constante. El germen de la Snack Culture estaba sembrado mucho antes desde luego (los estudios sobre la dieta tele- 


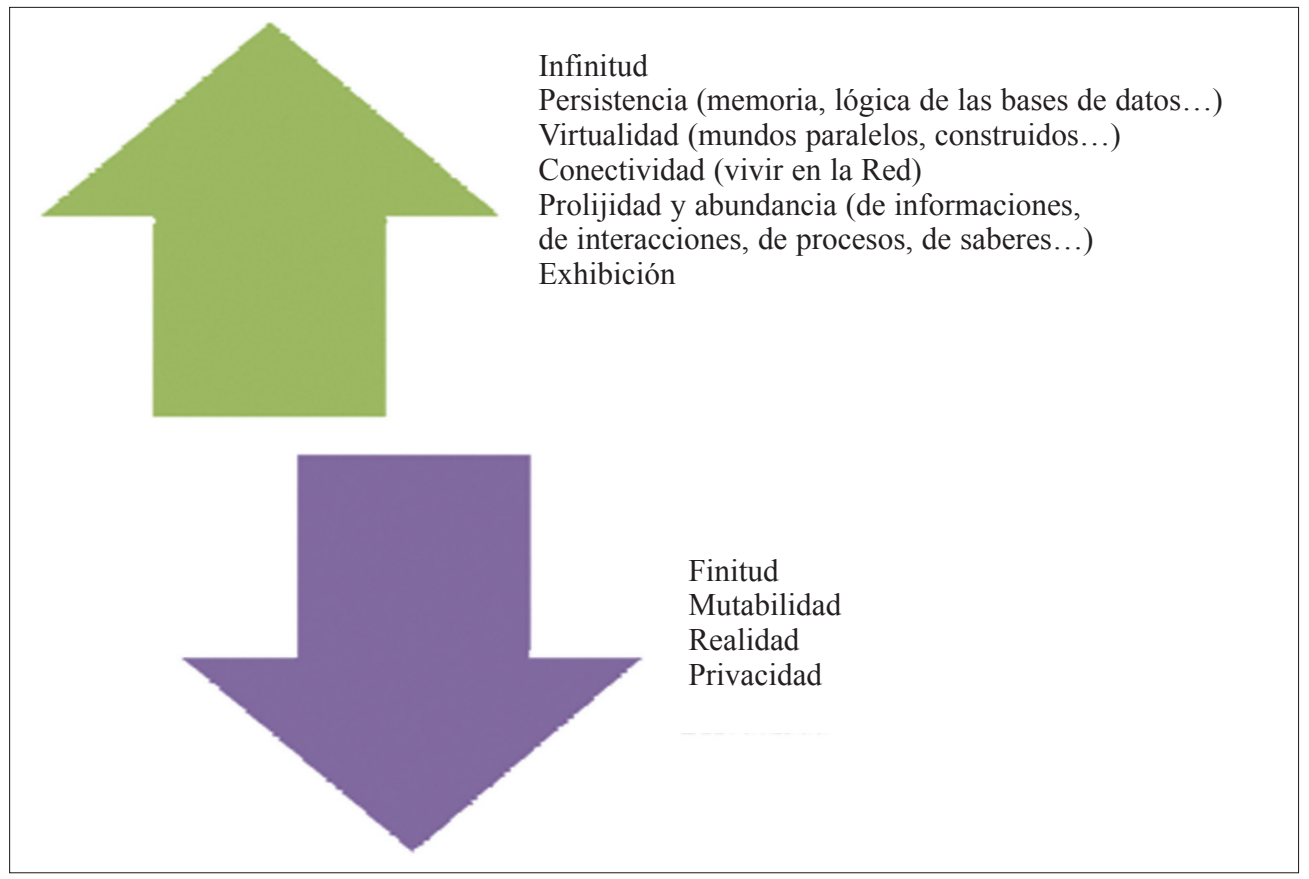

Figura 1.

Rasgos del Ciberespacio.

Fuente: Elaboración propia a partir de datos de Trejo (2006).

visiva mediada por el mando a distancia han aportado mucho en este campo) pero es evidente que este modelo cultural, también denominado transmodernidad, personismo, alude a un nuevo modelo, a una nueva era de consumo basada en la "frugalidad", en la rapidez, en un elogio a la "hiperactividad", en la inmediatez del reporte de la satisfacción, etc. Como indica Miller (2007): "Replace Nabisco with Apple, the Mini Oreo with the iPod nano, and you've got a blueprint for the current boom in what might be called snack-o-tainment".

Y esa hiperactividad, ese escópico bombardeo estimular, se multiplica con la presencia de todas las pantallas a un tiempo. Para tratar de explicar cómo nos adaptamos a ese contexto, la denominada Economía de la atención (Davenport \& Beck 2002, Goldhaber 1986, Lanham 2007) ha sentado algunos principios especialmente útiles. Nos ofrece un modelo explicativo del modelo económico que subyace en la producción y reparto en Internet. Toma la atención humana, de cada uno de nosotros, como valor de referencia. Se parte del enorme e inabarcable tamaño actual de la Red (information overload) (Gross 1964, Toffler 1981) y de la información contenida en ella, y de su crecimiento exponencial, y se contrapone a la capacidad que tenemos de atención las personas, que es constante (el cociente $\mathrm{n}^{\mathrm{o}}$. de Fuentes/Observadores disminuye). A partir de esto, y en el contexto de la satisfacción de las necesidades de usuario que realizan los servicios y contenidos digitales abiertos, se explica entre otras cuestiones la importancia creciente que 
poseen los servicios destinados a la gestión de la información; desde los buscadores, a los sistemas de recomendación, de filtrado de la información, etc. Cada vez tenemos menos tiempo (se genera una creciente escasez) y las necesidad de optimizarlo es mayor. Por eso, también crece la relevancia de las redes sociales, y entran en juego nuevas estrategias de gestión inteligente que se imbrican con el diseño de los contenidos y servicios digitales; el contenido, que aparece ahora como producto de valor añadido, se asocia a las acciones de comercialización en la Red. Nuevas competencias, en definitiva, que permitan a los usuarios "poner en práctica de forma integrada, en contextos diferentes, los conocimientos, habilidades y características de la personalidad adquiridas y/o desarrolladas" (GertrudixBarrio 2009: 58)

\section{INTELIGENCIA COLECTIVA, BAZARES Y "MEMES"}

Afirma Manovich (2006: 93) que la tecnología tiene una capacidad extraordinaria para modelar el tipo de producto cultural, su organización, sus géneros emergentes y sus contenidos. Así, las convergencias tecnológicas han activado no solo sus posibilidades de acción, sino también nuevas convergencias expresivas que son mediadas por los actores en cuanto que actúan de diversa forma si se trata del medio mismo o del medio en convergencia con otros (García García, 2006).

Planteamientos teóricos tan distanciados temporalmente como el del Teorema de Coase de 1932 (Coase 1988) o la teoría del estilo bazar de Raymond de 1998 (Raymond 2000) nos resultan especialmente útiles para analizar fenómenos de plena actualidad como la producción entre pares, o encontrar valiosas explicaciones para comprender el fundamento de los modelos de producción de los actuales servicios y contenidos digitales abiertos. Y es que, "la producción tipo bazar asigna de forma más eficiente el capital humano captado realmente, bajo sutiles condiciones de remuneración por incentivos indirectos de un conjunto muy numeroso, amplio y disperso de talentos. La potencia y facilidad de las redes hacen el resto, posibilitando el acceso y la comunicación en esta ad-hocracia virtual de nodos cooperantes" (Sáez Vacas 2004: 88)

Ahora, la construcción de los textos y discursos en la Red se realiza de forma cooperada. Textos y contenidos a los que los usuarios exigen un alto grado de calidad que se caracteriza por la fiabilidad, reutilización, intercambio, catalogación, reconocimiento y utilidad (Gértrudix Barrio et al, 2007) Los usuarios se han apropiado de los medios de producción y el usuario final forma parte de toda la red de suministros. La "generación digital" participa cada vez de forma más activa y es creadora de este nuevo medio de construcción cultural, desarrollando contenidos, diseñando webs personales y lanzando sus propias empresas innovadoras en Red.

Pero los "nativos digitales" no solo reciben y producen contenidos digitales, sino que forman parte de la retícula que vela por la gestión de la información en la Red, así como de las acciones de los internautas en las interrelaciones entre los distintos medios. El modelo de organización, gestión y etiquetado folksonómico de la infor- 
mación que facilita la Red a los usuarios, remite al pensamiento de Negroponte (1995) cuando al preguntarse si la naturaleza de un medio puede reproducirse en otro, decía: "La respuesta consiste en crear ordenadores, clasificar, seleccionar y manejar multimedia en beneficio propio; ordenadores que lean periódicos y miren la televisión por nosotros, y que actúen como editores cuando se lo pidamos", y se proyecta en los vaticinios que autores como Spivack (2007) o Castells (2009) han concedido a la web semántica.

Detrás de todo este movimiento subyace el concepto de inteligencia colectiva. Ligado a él, Don Tascopp y Anthony D. Williams (2007) utilizan el término Wikinomics para referirse a una nueva economía basada en la aportación de las multitudes inteligentes: "Wikinomics anuncia un cambio de paradigma en la colaboración entre grupos humanos. Gracias a internet, individuos que están más allá de las fronteras de las jerarquías tradicionales pueden innovar para producir contenidos".

Para abundar en el análisis de estos nuevos modos de producción en la Red, que se apalancan en las posibilidades que ofrecen los servicios digitales abiertos, interesa la integración que el concepto teórico y el desarrollo conceptual de la memética ha tenido en relación con las redes informacionales. La cristalización del concepto "meme" llevada a cabo por Richard Dawkins en 1976 (2000) refleja, en el ámbito de nuestro interés, "patrones contagiosos de información cultural que se transmiten de una mente a otra por medio de la selección, la infección y la replicación. Una idea o patrón de información no es un "meme" hasta que alguien lo replica, transmitiéndolo a otra persona, $\mathrm{y}$, la probabilidad de que un meme sea contagioso en un grupo está directamente relacionada con los valores, creencias y actividades de ese grupo" (Lankshear, 2008: 212) y constata el interés humano por los patrones, los procesos de decisión y las estructuras, contextos y actividades sociales.

El actual desarrollo de las redes facilita la propagación de "memes" digitales, irradiándose de forma viral gracias a los "espacios de afinidad" que han propiciado las herramientas sociales en la Web, explotando las características esenciales de los memes: a) la fidelidad, es decir la sencillez en el proceso de copiado, e identidad entre matriz y copia; b) la susceptibilidad, que ligada a la anterior, define el marco de aceptación que tiene un meme frente a otros; es decir, el grado de permeabilidad que poseen los sujetos o grupos para aceptar, interiorizar y propagar ese meme de forma privilegiada; c) la fecundidad, entendida como la velocidad de propagación de un meme. Los sistemas actuales de Red facilitan la radiación planetaria de un meme de forma prácticamente inmediata, lo que tiene una importancia capital en la forma de producir, consumir, intercambiar contenidos y servicios digitales abiertos. Pero la configuración de las redes sociales, entendidas como espacios de proximidad, amplia aún más dicha fecundidad al ofrecer a los memes vías de favorecidas de confianza; un contenido que proviene de una fuente conocida, del círculo próximo, tiene mayores posibilidades de ser atendida; y d) la longevidad, ya que la larga vida de un meme garantiza que su irradiación será mayor, alcanzando a un porcentaje más alto de la población.

Vistas sus características, y siguiendo a Lankshear y Knobel (2008), consideramos un meme en Internet como un concepto aplicado, y de pretensiones más modes- 
tas que su original "que describe las rápidas asunción y difusión de una determinada idea presentada como texto escrito, imagen, "cambio lingüístico" o cualquier otra unidad de material cultural"

Pero no faltan tampoco las aproximaciones críticas que como la Teoría crítica sobre la co-creación (Ritzer, 2006) ponen de relieve aspectos críticos tales como a quién pertenecen los datos que compartimos en la "nube", quién los explota y se beneficia de ellos, etc. Posturas que ponen de relieve el carácter indeterminado de los datos si carecen de un sentido que los vectoralice: "Para que la información deje de ser solo un conjunto de datos organizados (una granja de datos) y se convierta en conocimiento, es preciso el desarrollo y la actualización de estrategias criticas en los navegantes: selección, procesamiento, análisis, reflexión, reconstrucción, etc. que permitan apropiarse realmente del conocimiento que esa información es capaz de otorgar; un ejercicio global de inteligencia que permita aprehender y mejorar" (García García \& Gertrudix-Barrio, 2009: 14).

\section{UNA PRIMERA CARACTERIZACIÓN DE LOS SERVICIOS Y CONTENIDOS DIGITALES ABIERTOS}

Partimos del presupuesto de que el desarrollo de contenidos y servicios digitales abiertos en la web actual forma parte de un flujo, de una continuidad, y por tanto, aunque hagamos un ejercicio de análisis y de segmentación de esa realidad, los elementos que conforman ese flujo lejos de ser realidades aisladas, unívocas y con perímetros claramente definidos, son partículas que balizan en una especie de magma en sempiterno bullir: chocan unas contra otras, se permutan, cambian en el transcurso fluir, y configuran nuevas propuestas prácticamente a cada instante. Siendo así, es interesante considerar que el conjunto global conforma un suerte de discurso o, para ser más precisos, un malla discursiva, entendida tanto como un evento comunicativo de orden cultural, como un modelo de interacción.

Desde la perspectiva de los sujetos que operan estos contenidos y servicios digitales abiertos, y bajo la dimensión de las competencias que ponen en juego dentro del paradigma de las nuevas alfabetizaciones, esa participación en el discurso es plural y multidimensional: "Los alfabetismos son siempre mucho más e implican mucho más que la simple producción de textos. Son (también) contextos o pretextos para activar o refinar pertenencias a discursos con dimensiones tales como retroinformar, prestar apoyo, compartir conocimientos y pericia, explicar reglas, contar chistes, compadecer, hacer el trabajo propio, expresar opiniones, mostrar solidaridad, reflejar una afinidad, etcétera" (Gee, 2004).

Precisamente, para conocer los rasgos que definen esa malla discursiva, y para evaluar qué competencias se derivan de su uso, resulta de interés conocer la topografía, el mapa que se redibuja en la Red a través de estos entornos. Llegados a este punto, posiblemente por su carácter dinámico, resulta difícil encontrar un ejercicio teórico que, a día de hoy, haya propuesto un modelo clasificador, una taxonomía de los servicios y contenidos digitales abiertos que haya sido asumida como tal. Los intentos, numerosos eso sí, de fundar clasificaciones tienen hasta la fecha más el 
trazo de básicos mapas de ruta para exploradores intrépidos que una topografía calculada, debatida y contrastada. Cada uno da un paso a favor de obra, pero no disponemos aún de una vista de pájaro, del gran teatro del mundo.

Por eso, consideramos que la aportación que se realiza en este campo tiene especial valor; precisamente porque trata de contribuir a esa tarea cartográfica que, sin circular sobre la parábola de Borges, ha de interpretar una realidad diversa, amplia y móvil con el ánimo de establecer coordenadas de referencia, de situar cotas, de dibujar diagramas que sean capaces de ajustarse a la dinámica evolutiva de unos servicios y contenidos digitales abiertos que mutan como las costas al navegar.

Una parte importante de la teorización sobre la definición de los servicios y contenidos digitales se ha dirigido a establecer cuáles son las características que los definen. Aunque son muchas y diversas, hay un cierto consenso al establecer las siguientes.

El primero de ellos alude al concepto de sincretismo "digital". Tomando prestado el término del sistema filosófico, en el ámbito del desarrollo de servicios y contenidos digitales podemos hablar de un verdadero sincretismo digital por cuanto estos tienen, en la mayoría de los casos, a sumar capacidades y características cada vez más amplias y diversas, "fusionando" y recombinando las exitosas de sus competidores o, simplemente, integrando en ellas nuevas áreas y soluciones.

La hibridación es un proceso de combinación, de remezcla, de mestizaje, de recombinación... El término, por tanto, alude a una de las pautas básicas de la Cultura Remix (Remixología) (Latterell: 2009) y es una estrategia de muchos de los servicios que ofrece la Web social, así como una fórmula de innovación sobre la que explorar el recorrido de los nuevos medios. Por supuesto, la hibridación también establece, necesariamente, relaciones con la creatividad, pues se convierte en una herramienta al servicio de soluciones creativas.

La tecnología actual (AJAX, microformatos, APIs....) las nuevas lógicas de negocio (sistemas abiertos) y la semantización de la información de la Red (lógicas estructurales) posibilitan la emergencia de sistemas y contenidos basados en la reformulación. Desde los sistemas de sharing que proveen herramientas para la edición sencilla e inmediata, hasta los nuevos medios híbridos o semiautomatizados.

La remixación es tanto una lógica, un proceso como un producto de la hibridación. En los servicios y contenidos abiertos en la Red puede ser: a) de contenidos, como sucede con los media disponibles en los sistemas de sharing, o localizados mediante buscadores, directorios, etc. donde se remezclan elementos de nivel de agregación elemental; b) de "paquetes" web, mediante la recombinación de bloques estructurales de información integrados por conjuntos de medias, desde una noticia, a una web entera, y es el modelo que siguen, por ejemplo, los agregadores o los sistemas Webtop; c) de referencias o metainformaciones, en la medida que recombinan fuentes de información, no la información misma, aunque puedan utilizar parte de estas, como cabeceras, resúmenes, etc. Esta es la base de los sistemas de recomendación, de filtrado, los medios híbridos armados mediante algoritmos de selección (capas artificiales de edición, presentación...)

La personalización automatizada se soporta en la incorporación de semántica a los contenidos de la Red. El carácter semántico de los contenidos facilita su entrega y con- 
figuración personalizada en función del comportamiento de los usuarios (behaviour user) de sus preferencias, de la de sus círculos de proximidad en la Red, etc. Muchos sistemas acomodan la presentación de información que presentan a los internautas (desde los buscadores en sus resultados patrocinados, a los sistemas de recomendación automatizada como las radios online tales como Pandora, pasando por las redes sociales) bien en función de la información de perfil introducida por el propio usuario, bien mediante el registro y seguimiento de la actividad que este realiza.

La multimedialidad describe la conjunción y simultaneidad de diversos medios, como imágenes, sonidos y texto. El primer rasgo del multimedia que observamos es el combinatorio, la suma de cosas; de cosas que son... otros medios. El segundo, su adjetividad (su aplicabilidad a cosas y elementos que, en su contacto, se califican) tiene una vocación sustantiva; de la "cosa" multimedia progresamos hacia "el multimedia". El tercero, su complejidad; en esa suma de medios aparecen lenguajes y gramáticas complejas, formas de hacer, tecnologías y modos de aproximarse diversas. Retomando las ideas de Cotton y Oliver (1997) entendemos que lo "multimedia", en mayúsculas, es una instancia formal que toma naturaleza en la capacidad de crear experiencias interactivas hipermediales, es decir, de configurar procesos ricos en interactividad mediante la combinación de distintas sustancias expresivas y medios de comunicación en "los que se produce un cambio de roles: el autor pasa a ser auto-lector y viceversa, el lector es ahora lecto-autor" (García García: 2003)

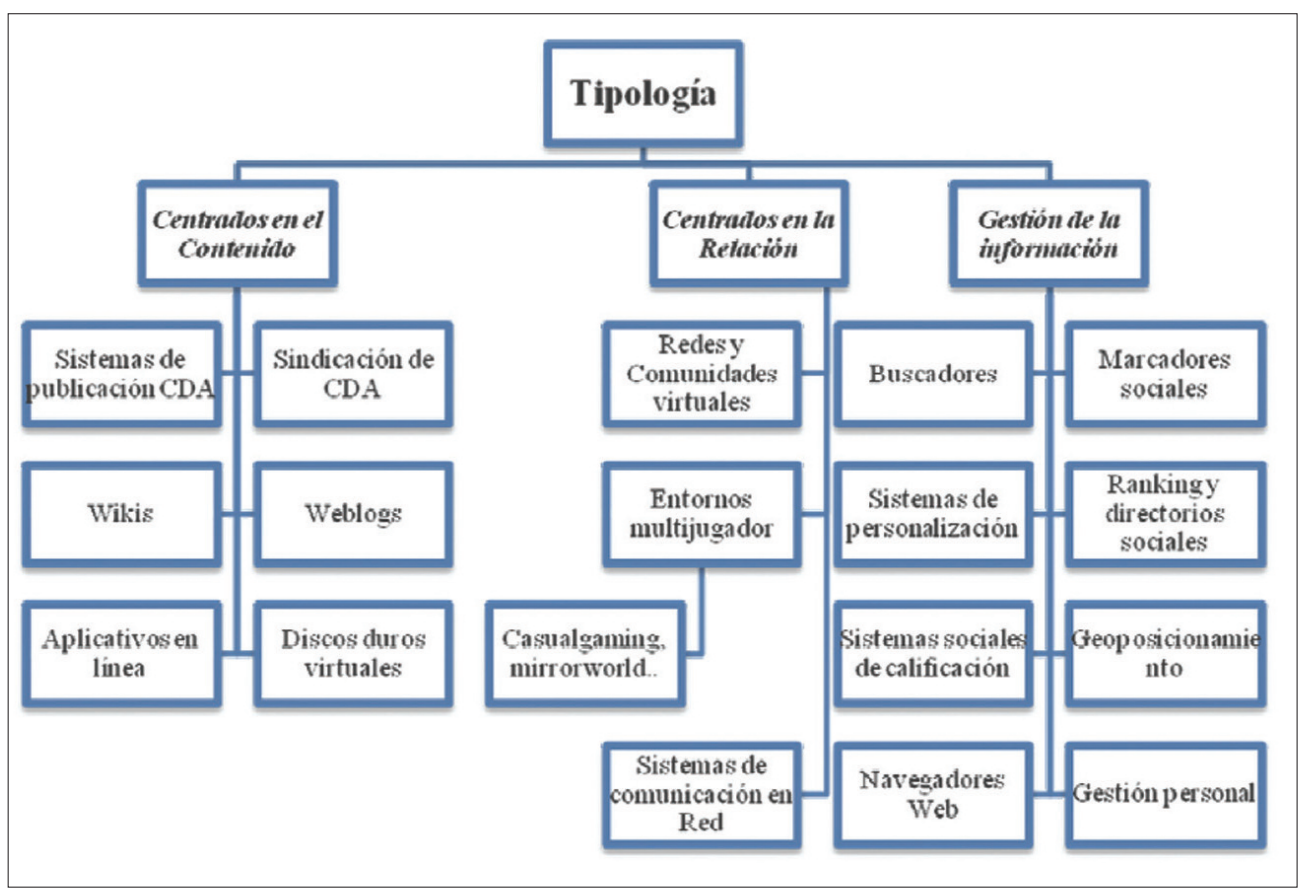

Figura 2.

Tipología de Servicios Digitales Abiertos. Fuente: Elaboración propia 
La diversidad y continua emergencia de CDAs y SDAs hace necesario, para comprender la dimensión y profundidad de este fenómeno, realizar una tipología de contenidos y servicios digitales abiertos, es decir, una organización sistemática y jerárquica de contenidos y servicios digitales abiertos que siga un determinado paradigma clasificatorio. De forma tentativa, el gráfico siguiente resume una propuesta de SDAs que utiliza un eje axiomático basado en la funcionalidad "ontológica" del servicio y/o contenido, es decir, la razón esencial que le da sentido y el elemento sustancial que hace que sea utilizado por los usuarios, segmentando los contenidos y servicios digitales abiertos en tres grandes bloques: a) Centrados en el contenido; b) Centrados en la Relación; c) Centrados en la Gestión de Información.

Temáticamente se atienden, de inicio, a todos los servicios y contenidos (sistemas facilitadores) pues forman parte de un Censo de servicios y contenidos digitales que se ha realizado en los últimos dos años y que se encuentra disponible en la URL http://delicious.com/socmedia

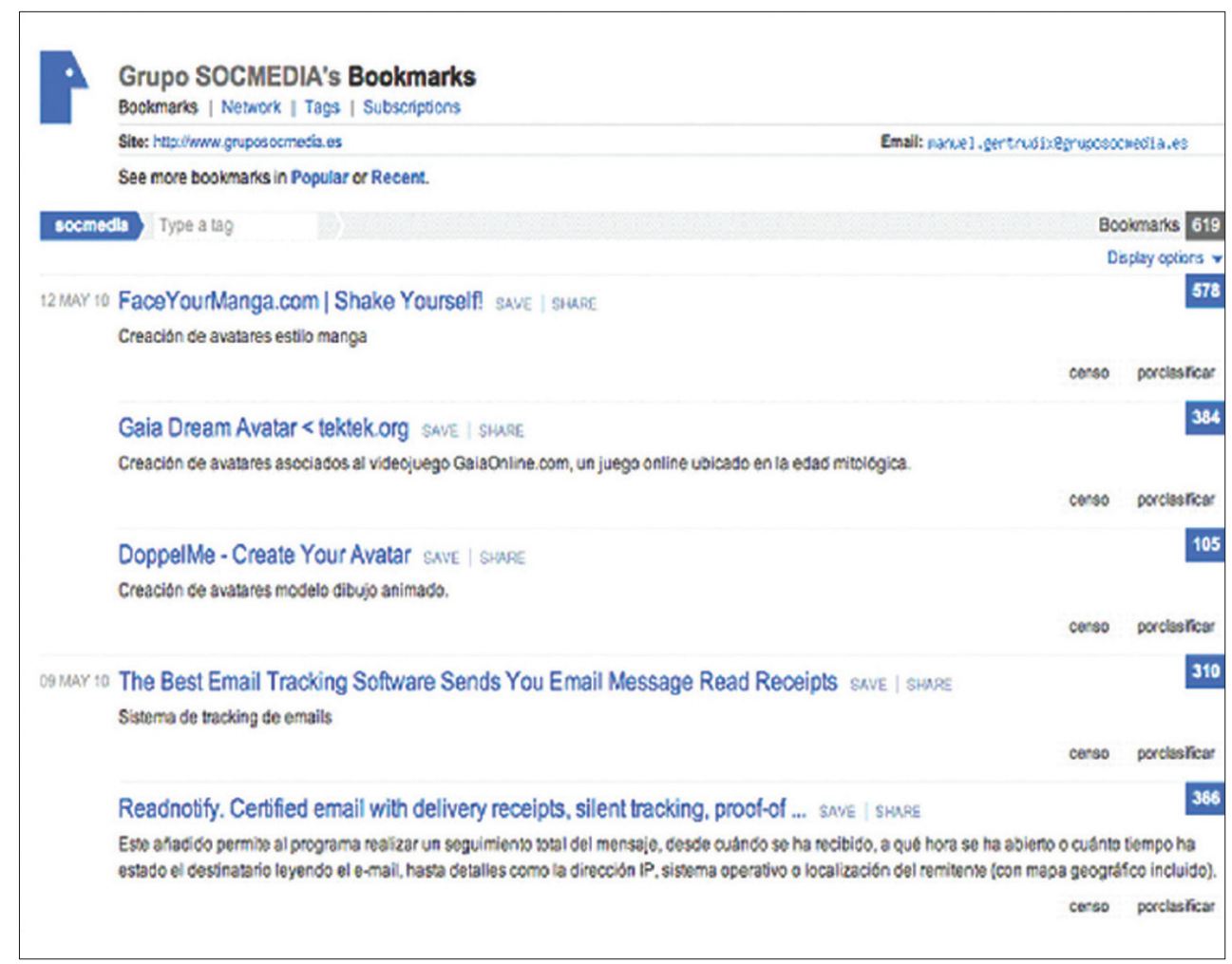

Figura 3

Censo de Servicios y Contenidos Digitales Abiertos.

Fuente: Elaboración propia. Consultado el 10-01-2011. 


\section{PRIMERAS CONCLUSIONES}

Los contenidos y servicios digitales abiertos constituyen, actualmente, una amplia gama de productos Web que integran, bajo ciertos rasgos genéricos, propiedades diferenciales y específicas de lo que se conoce como Web 2.0 o Web social.

La lógica evolutiva de la Red (cuando aún se consolida el fenómeno de la 2.0, se alumbra ya el escenario de una Web 3.0 basada en interfaces 3D, o realmente semántica) hace que el escenario de análisis de los contenidos y servicios digitales abiertos sea muy volátil, por lo que las propiedades observadas se categorizan en grandes ámbitos funcionales que permitan su seguimiento longitudinal.

El infoentorno (ciberespacio, ciberinfinito...) posee ciertas propiedades que, aún con las diferencias de aproximación de diferentes escuelas o autores, son características: desigualdad, distalidad, exuberancia... Dichas propiedades, necesariamente, osmotizan los productos que se generan en la Red, por lo que los contenidos y servicios digitales recuperan y despliegan parte de ellas.

Existen diferentes ideologías, "mentalidades" o imaginarios que construyen la Red y "mediatizan" su evolución. Frente al modelo intervencionista se posiciona el modelo libre de la "frontera electrónica". Ante los modelos de distribución controlada (sistema de construcción de las catedrales, de la lógica de las patentes y el copyright), los defensores de la Cultura Libre y los promotores de las licencias de código abierto y Copyleft.

Estamos asistiendo a la consolidación de nuevos modelos culturales basados en el tiempo como producto de valor. La escasez de tiempo favorece la emergencia de una economía de la atencióny el desarrollo de modelos de consumo frugales, a veces fútiles, en cualquier caso, en estado de emergencia; una cultura snack que cambia los sistemas de producción de información, las narrativas y los discursos.

La Red se articula como un sistema en el que lo colectivo penetra en lo individual. Los contenidos ya no son entes cerrados, objetos finitos y finalizados, sino contenedores abiertos, en constante reelaboración.

Los flujos de producción, modificación, intercambio y remixación de contenidos responden a lógicas propias de entes colectivos que irradian una suerte de inteligencia colectiva y que generan e irradian un "acervo memético en la Red"

No existe, a día de hoy, un modelo clasificador, una taxonomía de los servicios y contenidos digitales abiertos que haya sido asumida como tal. Los intentos, numerosos eso sí, de fundar clasificaciones tienen hasta la fecha más el trazo de básicos mapas de ruta para exploradores intrépidos que una topografía calculada, debatida y contrastada. 


\section{REFERENCIAS BIBLIOGRÁFICAS}

AAVV. (2010): Color IURIS. En línea. http://www.coloriuris.net/ Consultada el 18-12-2010.

AAVV. (2010): Creative Commons España. En línea. http://es.creativecommons.org/ Consultada el 18-12-2010.

BeLl, D. (2007): Theorists, Cybercultures. New York: Routledge.

Benkler, Y. (2006): The Wealth of Network: How Social Production Transforms Markets and Freedom. En línea. Yale University Press:

http://www.benkler.org/Benkler_Wealth_Of_Networks.pdf Consultada el 22-12-2010.

CARR, N. (2008): El gran interruptor. Barcelona: Editorial Deusto.

Castells, M. (2000): The Rise of the Network Society: The Information Age: Economy, Society, and Culture Volume I. Oxford: Wiley-Blackwell.

CoAse, R. H. (1988): The Firm, the Market and the Law. Chicago: The University of Chicago Press.

Cotton, B., \& Oliver, R. (1997): Understanding Hypermedia 2.000: Multimedia Origins, Internet Futures. London: Phaidon.

Davenport, T., \& Beck, J. (2002): The Attention Economy: Understanding the New Currency of Business. Harvard: Harvard Business Press.

Dawkins, R. (2000): The Meme Machine. Oxford: Oxford University Press.

EChevarría, J. (2000): Telépolis. Madrid: Destino.

GARCÍA GARCíA, F. (2006): "De la convergencia tecnológica a la convergencia comunicativa en la educación y el progreso". Madrid: Revista ICONO14, 3-18.

- (2003): "La narrativa hipermedia aplicada a la educación". Madrid: Red Digital, 8-22.

García García, F., \& GertrudiX-Barrio, M. (2009): “El Mare Nostrum Digital: Mito, ideología y realidad de un imaginario sociotécnico". Madrid: Revista ICONO14 nº. 12, 730.

GeE, J. P. (2004): Good Video Games and Good Learning. New York: Peter Lang.

- (2004): Situated Language and Learning: A Critique of Tradicional Schooling. London: Routledge.

GertrudiX-BARrio, F. (2009): "Internet como espacio de adquisición de competencias". Madrid: Revista ICONO14 n'. 12, 54-72.

GÉrtrudiX BARrio, M., \& AL, E. (2007): "Design and development of Digital Educational Content”. En B. Fernández-Manjón, \& J. M. Sánchez-Pérez, Computers and Education: e-learning from theory to practice. Dordrech: Springer.

Gibson, W. (2004): Neuromante. Barcelona: Minotauro.

Goldhaber, M. (1986): Reinventing Technology. London: Routledge \& Kegan Paul Books Ltd.

Gross, B. (1964): The Managing of Organizations: The Administrative Struggle. New York: Free Press of Glencoe.

Guengerich, S. (2010): Think Global, Act Glocal. USA: Lulu.com.

Hughes, K. (1995): From webspace to cyberspace. Menlo Park: Enterprise Integration Technologies.

Lanham, R. (2007): The Economics of Attention: Style and Substance in the Age of Information. Chicago: University Of Chicago Press.

LANKSHEAR, C. K. (2008): Nuevos alfabetismos. Su práctica cotidiana y el aprendizaje en el aula. Madrid: Morata. 
Latterell, C. G. (2009): ReMix: Reading and Composing Culture. New York: Bedford/St. Martin's.

Negroponte, N. (1995): Ser Digital. Buenos Aires: Atlantida.

MacLuhan, M. (1969): El medio es el mensaje: un inventario de efectos. Buenos Aires: Paidós.

Manovich, L. (2006): El Lenguaje de Los Nuevos Medios de Comunicacion. Barcelona: Paidós Ibérica.

Miller, N. (2007): “Manifesto for a New Age”. En línea. Wired:

http://www.wired.com/wired/archive/15.03/snackminifesto.html Consultada el 12-122010

Pisani, F., Piotet, D., \& Delclos, T. (2009): La alquimia de las multitudes: Como la web esta cambiando el mundo. Barcelona: Paidos Iberica Ediciones.

RAYMOnd, E. S. (2000): Cathedral and the Bazaar. USA: Snowballpublishing.com.

Ritzer, G. (2006): Contemporary Sociological Theory and Its Classical Roots: The Basics. London: McGraw-Hill Humanities.

SpIVACK, N. (2007): Minding the Planet. En línea. http://www.novaspivack.com Consultada el 8-12-2010.

Sonvilla-WeIss, S. (2010): Mashup Cultures. New York: Springer Vienna Architecture.

Tapscott, D., \& Williams, A. (2007): Wikinomics: How Mass Collaboration Changes Everything. New York: Portfolio Trade.

Toffler, A. (1981): La tercera ola. Barcelona: Plaza \& Janes Editores .

Trejo Delabre, R. (2006): Viviendo en el Aleph. Barcelona: Gedisa.

Verdú, V. (2007): Yo y Tu, Objetos de lujo. El personismo: la primera revolución cultural del siglo XXI. Barcelona: Debolsillo.

WiENER, N. (1985): Cibernética o el control y comunicación en animales y máquinas. Barcelona: Tusquets.

\section{RESUMEN}

Se realiza una aproximación a la naturaleza y características de los servicios y contenidos digitales abiertos en la red desde su análisis como componentes del sistema actual de medios. Se contextualizan su emergencia en el ecosistema sociocultural actual y de despliegue tecnológico en el que se desarrollan, caracterizándolos por sus rasgos esenciales, y proponiendo un modelo taxonómico que ayude a comprender el alcance de este fenómeno desde las Ciencias de la Comunicación. Para ello, se ha efectuado una revisión documental para establecer las principales aproximaciones teóricas realizadas así como las categorías claves para su análisis, se ha inventariado la oferta de servicios y contenidos digitales abiertos modelo, y se ha realizado una propuesta clasificatoria. Los resultados ofrecen una topografía inicial de la cartera de servicios disponibles organizados según criterios funcionales y operativos.

Palabras clave: Contenidos digitales abiertos, servicios digitales abiertos, nativos digitales, cultura remix, infoentorno, economía de la atención. 


\title{
RÉSUMÉ
}

L'article analyse la nature et les contenus des services et contenus digitales ouverts dans l'Internet en tant que components su système actuel des médias. Il étudie leur emergeance dans l'ecosystème socioculturel actuel et observe leurs traits essentiels, en proposant un modèle taxonomique pour comprendre le phénomène dans le contexte des Sciences de la Communication. On a effectué une révision documental des theories ainsi que des categories, un inventaire des modèles des continus ouverts et on a proposé une clasification. Les résultats offrent une topographie des services disponibles organisés par critères fonctionnels et operatifs.

Mots clé: Contenus digitaux ouverts, services digitaux ouverts, natifs digitaux, cultura remix, infoenvironement, économie de l'attention.

\begin{abstract}
This research analyzes the Open Digital Services and Open Digital Content. We study their characteristics and components as part of the current system of media on the Web.It explains the importance they have in the socio-cultural ecosystem and current technology, and is characterized by its essential features, and proposing taxonomic model that helps understand the scope of this phenomenon from the Communication Sciences. To do this, first there has been a literature review to establish the main theoretical approaches taken as well as key categories for analysis. Secondly, it has made an inventory of the supply of services and open digital content, and has made a proposal for classification. The results provide an initial topography of the portfolio of available services organized according to their functionality and operability.
\end{abstract}

Key words: Open Digital Content, Open Digital Services, Digital Natives, Remix Culture, Attention Economy. 